\title{
Improved Binding Activity of Antibodies against Major Histocompatibility Complex Class I Chain-Related Gene A by Phage Display Technology for Cancer-Targeted Therapy
}

\author{
Achara Phumyen, ${ }^{1,2,3}$ Amonrat Jumnainsong, ${ }^{3}$ and Chanvit Leelayuwat ${ }^{2,3,4}$ \\ ${ }^{1}$ Biomedical Sciences Program, Graduate School, Khon Kaen University, Khon Kaen 40002, Thailand \\ ${ }^{2}$ Specific Health Problem of Grater Maekong Subregion (SHeP-GMS), Khon Kaen University, Khon Kaen 40002, Thailand \\ ${ }^{3}$ The Centre for Research and Development of Medical Diagnostic Laboratories (CMDL), Faculty of Associated Medical Sciences, \\ Khon Kaen University, Khon Kaen 40002, Thailand \\ ${ }^{4}$ Department of Clinical Immunology and Transfusion Sciences, Faculty of Associated Medical Sciences, Khon Kaen University, \\ Khon Kaen 40002, Thailand
}

Correspondence should be addressed to Chanvit Leelayuwat, chanvit@kku.ac.th

Received 20 July 2012; Accepted 14 September 2012

Academic Editor: Dirk Saerens

Copyright (c) 2012 Achara Phumyen et al. This is an open access article distributed under the Creative Commons Attribution License, which permits unrestricted use, distribution, and reproduction in any medium, provided the original work is properly cited.

\begin{abstract}
Major histocompatibility complex class I chain-related gene A (MICA) is an NKG2D ligand that is over-expressed under cellular stress including cancer transformation and viral infection. High expression of MICA in cancer tissues or patients' sera is useful for prognostic or follow-up markers in cancer patients. In this study, phage display technology was employed to improve antigenbinding activities of anti-MICA monoclonal antibodies (WW2G8, WW6B7, and WW9B8). The 12 amino acid residues in the complementarity determining regions (CDRs) on the V domain of the heavy chain CDR3 (HCDR3) of these anti-MICA antibodies were modified by PCR-random mutagenesis, and phages displaying mutated anti-MICA Fab were constructed. After seven rounds of panning, five clones of phages displaying mutant anti-MICA Fab which exhibited 3-7-folds higher antigen-binding activities were isolated. Two clones of the mutants (phage-displayed mutant Fab WW9B8.1 and phage-displayed mutant Fab WW9B8.21) were confirmed to have antigen-binding specificity for cell surface MICA proteins by flow cytometry. These phage clones are able to recognize MICA in a native form according to positive results obtained by indirect ELISA and flow cytometry. Thus, these phage particles could be potentially used for further development of nanomedicine specifically targeting cancer cells expressing MICA proteins.
\end{abstract}

\section{Introduction}

In human, NKG2D ligands consist of two families; the MHCclass-I- chain-related proteins (MICA and MICB) $[1,2]$ and the cytomegalovirus UL16-binding protein family (ULBP16) or retinoic acid early transcript 1 (RAET1E, G, H, I, $\mathrm{N}$, and RAET1L) $[3,4]$ which are polymorphic [5-7]. The signaling through NKG2D engagement to its ligands requires the adaptor protein DAP10 in human or DAP10/12 in mice, forming a hexameric complex on the cell membrane $[8,9]$. The NKG2D ligands are mostly not expressed on normal cells or expressed at very low levels on particular cells at particular conditions but are upregulated on cells under stress such as malignant cells or bacterial/viral infected cells and have been linked with autoimmune diseases [10-13]. Thus, the expression of NKG2D ligands is important in immune responses $[14,15]$.

At present, monoclonal antibodies (mAbs) are breakthrough in medicine and are effective products for diagnostic, monitoring, and therapeutic of cancers, infections, and other diseases. In case of cancer, many kinds of cancer cells are over expressing NKG2D ligands, but they can escape from recognition and destruction by $\mathrm{T}$ cells or NK cells of the immune system by shedding the ligands as a soluble form such as soluble MIC. These molecules can be detected in blood samples from patients. Soluble ligands can 
downregulate effective killing of tumor cells [16]. The MIC shedding in cancer renders a reduced or low expression of MIC on cell surface. Therefore, the high affinity antibodies are required for prognostics and therapeutics in cancer patients.

Previously, we have generated several monoclonal antibodies against MICA [17]. In order to improve the binding activities against MICA, these antibodies were cloned and displayed on filamentous bacteriophages in this study. In addition, affinity maturation of antibodies expressed on phages was performed by PCR-random mutagenesis at complementarity determining regions (CDRs) on the $\mathrm{V}$ domain of the heavy chain CDR3 (HCDR3). This process has produced clones with high anti-MICA activities which have been characterized. These clones would have high potential to develop targeted therapy against cancer cells expressing MICA.

\section{Material and Method}

2.1. Bacterial Strains. The Escherichia coli strain XL1Blue $\mathrm{MRF}^{\prime}(\Delta(m c r A) 183 \Delta($ mcrCB-hsdSMR-mrr $) 173$ endA1 supE44 thi-1 recA1 gyrA96 relA1 lac $\left[\mathrm{F}^{\prime}\right.$ proAB lacI $^{\mathrm{q}} \mathrm{Z} \Delta \mathrm{M} 15$ $\left.\operatorname{Tn} 10\left(\mathrm{Tet}^{\mathrm{r}}\right)\right]$ (Stratagene, USA) was used as the host for the preparation of phagemids, pComb3H-SS, kindly provided by Dr Barbas III [18] and as host for the helper bacteriophage, VCSM13 (GE healthcare Biosciences, NJ, USA).

2.2. Plasmids. The pGEM-T easy vector (Promega, USA) was used for cloning of PCR products, and the pComb3H-SS phagemid vector was used for the Fab displayed filamentous bacteriophage system.

2.3. cDNA from Anti-MICA Monoclones. Anti-MICA monoclones; WW2G8, WW6B7, WW9B8 were previously generated [17]. They were all IgG1 with kappa chain. Total RNA and cDNA were extracted and synthesized by oligo dT and random hexamer primers according to manufacturer's instructions using RNA extraction and cDNA synthesis kits (Promega, USA). These cDNA samples were used as templates for further PCR amplifications and cloning of heavy and light chains to phagemid, pComb3H-SS.

2.4. Construction of Fab from Anti-MICA Monoclonal Antibodies. In order to construct anti-MIC Fab, the $\mathrm{VH}$ and VL genes were amplified by PCR from cDNA of anti-MIC monoclones(WW2G8, WW6B7, and WW9B8). The heavy chain reverse primer; 5' A G G C T TACTAGTA C A A T C C C T G G G C A C A A T-3' (SpeI site is underlined) and the 3 heavy-chain variable forward primers; 5' AGGTCCAGCTGCTCGAGTCTGG3', 5' AGGTCCAACTGCTCGAGTCTGG-3', and 5' AGGTCCAACTGCTCGAGTCAGG-3' with XhoI sites underlined were used to amplify $\mathrm{VH}$. The kappa lightchain reverse primer; 5' GCGCCGTCTAGAATTAACACTCATTCCTGTTGAA-3' (XbaI site is underlined) and the 5 light-chain variable forward primers; $5^{\prime}$ CCA
GTTCCGAGCTCGTGCTCACCCAGTCTCCA-3', 5' CCAGTTCCGAGCTCCAGATGACCCAGTCTCCA-3', 5' CCAGATGTGAGCTCGTGATGACCCAGACTCCA-3', 5' CCAGATGTGAGCTCGTCATGACCCAGTCTCCA-3' ${ }^{\prime}$, and 5' CCAGTTCCGAGCTCGTGATGACACAGTCTCCA-3' with SacI sites underlined were used to amplify the VL gene. The resulting PCR products were first cloned into pGEM-T easy vector and then into pComb3H-SS phagemid vector with heavy chain as a fusion molecule with pIII [18]. After cloning and phage rescue, all clones were tested for binding with MICA by biopanning and indirect ELISA (described below). Direct sequencing of the phage clones to obtained heavy and light chain sequences was performed by 1st BASE laboratories Sdn Bhd., Malaysia, using pComb3H-SS specific forward primer: pC3HSS2575s (VL): 5'AAGACAGCTATCGCGATTGCAG3' [19], pelseq (VH): 5'ACCTATTGCCTACGGCAGCCG3', and pComb3H-SS-specific reverse primer: kpel (VL): 5'CGGCTGCCGTAGGCAATAGGT 3', gback (VH): 5'GCCCCCTTATTAGCGTTTGCCATC as sequencing primers. The sequences were analyzed using BLAST (IMGT, the international ImMunoGeneTics information system http://www.imgt.org/).

\subsection{Construction of Phage-Displayed Fab Carrying HCDR3} Mutants. PCR-random mutagenesis was performed to modify the complementarity determining regions (CDRs) on the $\mathrm{V}$ domain of the heavy chain CDR3 (HCDR3) of the clones using mutagenic primers (Table 1 ) as previously described [20], and mutated Fab displayed phages were reconstructed. These primers were designed from the clones validated by direct sequencing to randomize the 12 amino acid residues of HCDR3 region of anti-MICA antibodies by avoiding stop codons.

2.6. Production of-Anti-MICA Fab Displayed and AntiMICA-HCDR3 Mutant Fab Displayed Phages. To produce Fab displayed phages, XL-1Blue cells harboring phagemids were grown to an OD of 600 in $50 \mathrm{~mL}$ LB broth medium containing $50 \mu \mathrm{g} / \mathrm{mL}$ carbenicillin (carb) and $10 \mu \mathrm{g} / \mathrm{mL}$ tetracycline (Tet) and incubated at $37^{\circ} \mathrm{C}$ overnight on shaker. Following, $10^{12}$ plaque forming units ( $\mathrm{pfu}$ ) of helper phages, VCSM13, were added to the culture and incubated for $2 \mathrm{hr}$ at $37^{\circ} \mathrm{C}$. After that $70 \mu \mathrm{g} / \mathrm{mL}$ kanamycin was added to the culture and incubated on a shaker overnight at $37^{\circ} \mathrm{C}$. After centrifugation at 4,000 rpm for $15 \mathrm{~min}$, the supernatant carrying phages was transferred to a clean bottle and $4 \%$ $(\mathrm{w} / \mathrm{v})$ PEG-8000 and $3 \%(\mathrm{w} / \mathrm{v}) \mathrm{NaCl}$ were added, then, placed on shaker for $5 \mathrm{~min}$ to dissolve and precipitated phage on ice for $30 \mathrm{~min}$. The phage particles were isolated by centrifugation at $9,000 \mathrm{rpm}$ for $20 \mathrm{~min}$ at $4^{\circ} \mathrm{C}$ by discarding supernatant and allowing the bottle to drain on paper towel for $10 \mathrm{~min}$ to remove as much PEG solution as possible. The pellet, then, was resuspended in TBS/1\% BSA, and the solution was centrifuged at $14,000 \mathrm{rpm}$ for $5 \mathrm{~min}$. After centrifugation, the resulting supernatant containing the anti-MIC Fab displayed phages was stored at $4^{\circ} \mathrm{C}$ until used. 
TABLE 1: Primers for PCR-random mutation of HCDR3 region of anti-MICA antibodies.

\begin{tabular}{|c|c|c|c|}
\hline & Forward primers & Reverse primers & $\begin{array}{l}\text { Size of } \\
\text { product } \\
\text { (bp) }\end{array}$ \\
\hline (1) anti-MICA: WW2G8 & $\begin{array}{l}5^{\prime}-\text { TAT TgT NNY NNY NNY NNY NNY } \\
\text { NNY NNY NNY NNY NNY NNY NNY } \\
\text { Tgg ggT-3' }\end{array}$ & $\begin{array}{l}\text { 5'-ACC CCA RNN RNN RNN RNN RNN } \\
\text { RNN RNN RNN RNN RNN RNN RNN } \\
\text { ACA ATA-3' }\end{array}$ & $5000 \mathrm{bp}$ \\
\hline (2) anti-MICA: WW6B7 & $\begin{array}{l}5^{\prime}-\text { TAC TgT NNY NNY NNY NNY NNY } \\
\text { NNY NNY NNY NNY NNY NNY NNY } \\
\text { NNY NNY Tgg ggC-3' }\end{array}$ & $\begin{array}{l}5^{\prime} \text { - gCC CCA RNN RNN RNN RNN RNN } \\
\text { RNN RNN RNN RNN RNN RNN RNN } \\
\underline{\text { RNN RNN ACA gTA-3' }}\end{array}$ & $5000 \mathrm{bp}$ \\
\hline (3) anti-MICA: WW9B8 & $\begin{array}{l}5^{\prime}-\text { TAT TgT NNY NNY NNY NNY NNY } \\
\text { NNY NNY NNY NNY NNY NNY NNY } \\
\text { Tgg ggT-3' }\end{array}$ & $\begin{array}{l}\text { 5'-ACC CCA RNN RNN RNN RNN RNN } \\
\text { RNN RNN RNN RNN RNN RNN RNN } \\
\text { ACA ATA-3' }\end{array}$ & $5000 \mathrm{bp}$ \\
\hline
\end{tabular}

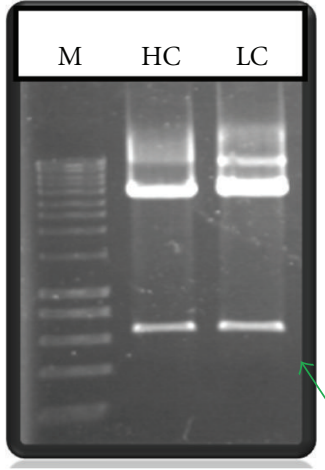

(a) WW2G8

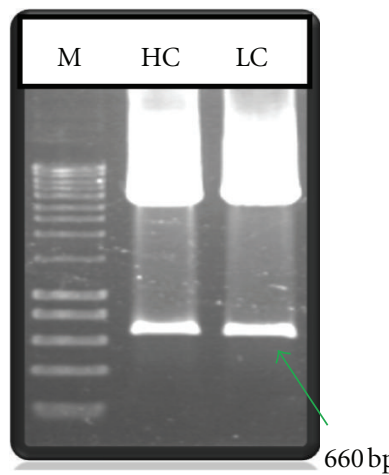

(b) WW6B7

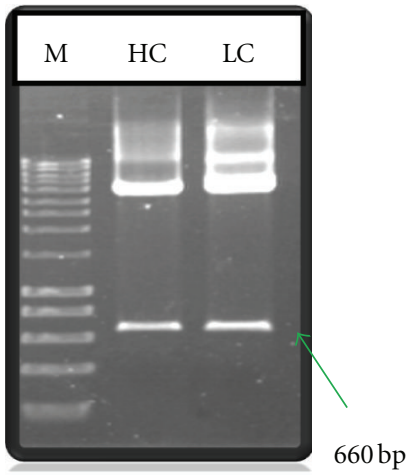

(c) WW9B8

FIGURE 1: Cloning of heavy and light chains into phagemids. XhoI/SpeI restricted heavy chain and XbaI/SacI restricted light chain inserted products of (a) WW2G8, (b) WW6B7, and (c) WW9B8 from phagemids were shown. The inserts of 660 bp were obtained for both heavy and light chains. (HC: heavy chain, LC: light chain, M: DNA marker).

2.7. Selection of Phage-Displayed Fab and Phage-Displayed Fab Carrying HCDR3 Mutants against MICA by Biopanning. An ELISA plate was coated with MICA antigens prepared as previously described [17] using $10 \mu \mathrm{g}, 5 \mu \mathrm{g}$, and $0.5 \mu \mathrm{g}$ for the first, second, and third panning, respectively, in coating buffer (bicarbonate $\mathrm{pH} 9.6$ ) overnight at $4^{\circ} \mathrm{C}$. The coating may also be performed at $37^{\circ} \mathrm{C}$ for $1 \mathrm{hr}$. Then, the plate was washed 2 times with TBS/0.5\% Tween (TBST) and blocked by filling the wells completely with $5 \%$ skim milk in TBS for $1 \mathrm{hr}$ at room temperature (RT). Following, phage-displayed Fab suspension containing $10^{12} \mathrm{pfu}$ in a total of $100 \mu \mathrm{L}$ was added to each well and incubated for $2 \mathrm{hr}$ at RT, then unbound phages were removed, and the plate was washed with TBS/0.5\% Tween (TBST) by vigorously pipetting up and down and waited for $5 \mathrm{~min}$ before removing TBST. In the first round, after unbound phages were removed, then the plate was washed once with TBST. The plate was washed 5 times in the 2 nd round and ten times in the $3 \mathrm{rd}$ and subsequent rounds. For affinity maturation, biopanning of up to ten rounds was performed. Bound phages were eluted by adding $50 \mu \mathrm{L}$ of elution buffer $(0.1 \mathrm{M} \mathrm{HCl}$ adjusted with glycine to $\mathrm{pH} 2.2$ with BSA $1 \mathrm{mg} / \mathrm{mL}$ ) per well and incubated for $10 \mathrm{~min}$ at RT, followed by pipetting up and down vigorously. The eluate was removed and neutralized with $3 \mu \mathrm{L}$ of $2 \mathrm{M}$ Tris-base per $50 \mu \mathrm{L}$ of eluate [18].

\subsection{Characterization of Phage-Display Anti-MICA Fab by Indirect ELISA and Flow Cytometry}

2.8.1. Indirect ELISA. ELISA wells coated with soluble MICA antigens $(5 \mu \mathrm{g})$ were incubated with $10^{8}$ or $10^{12}$ pfu phagedisplay anti-MICA Fab suspension in a total of $100 \mu \mathrm{L}$ for $2 \mathrm{hr}$ at RT. Following three washes with TBST, bound phages were stained with a $1: 5000$ diluted horseradish peroxidaseconjugated mouse anti-M13 monoclonal antibody (GE healthcare Biosciences, NJ, USA) for $1 \mathrm{hr}$ at RT followed by three washes. Bound phages were detected using 3,3',5,5'tetramethylbenzidine (TMB) (KPL, Gaithersburg, MD) and measured as optical density absorbance (OD), which was determined at $450 \mathrm{~nm}$.

2.8.2. Flow Cytometry. $293 \mathrm{~T}$ cells $\left(2 \times 10^{5}\right.$ cells $)$ expressing MICA were incubated with $100 \mu \mathrm{L}$ of phage-display antiMICA Fab suspension $\left(10^{11} \mathrm{pfu}\right)$ for $1 \mathrm{hr}$ on ice. The cells were washed with $1 \%$ bovine serum albumin in 


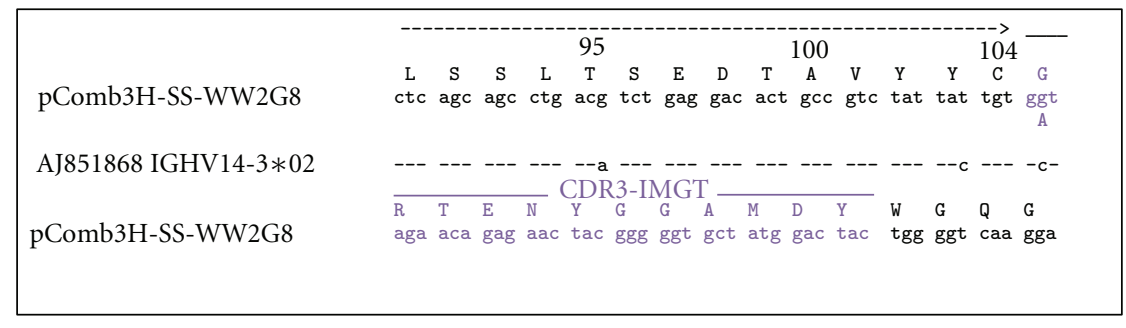

(a)

\begin{tabular}{|c|c|c|c|c|c|c|c|c|c|c|c|c|c|c|c|}
\hline \multirow[b]{3}{*}{ pComb3H- SS-WW6B7 } & \multicolumn{15}{|c|}{ 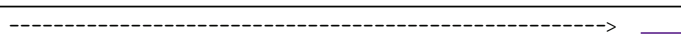 } \\
\hline & \multirow[b]{2}{*}{$\begin{array}{c}\mathrm{L} \\
\mathrm{ctc}\end{array}$} & \multirow[b]{2}{*}{$\begin{array}{c}\mathrm{S} \\
\mathrm{agc}\end{array}$} & \multirow[b]{2}{*}{$\begin{array}{c}\mathrm{S} \\
\mathrm{agc}\end{array}$} & \multicolumn{3}{|c|}{95} & \multirow[b]{2}{*}{$\begin{array}{c}\text { E } \\
\text { gag }\end{array}$} & \multirow[b]{2}{*}{$\begin{array}{c}\text { D } \\
\text { gac }\end{array}$} & \multicolumn{3}{|c|}{100} & \multirow[b]{2}{*}{$\begin{array}{l}Y \\
\text { tat }\end{array}$} & \multicolumn{3}{|c|}{104} \\
\hline & & & & $\begin{array}{c}\mathrm{L} \\
\operatorname{ctg}\end{array}$ & $\begin{array}{c}\mathrm{T} \\
\mathrm{aca}\end{array}$ & $\begin{array}{c}S \\
t c t\end{array}$ & & & $\begin{array}{c}\mathrm{T} \\
\text { act }\end{array}$ & $\begin{array}{c}\mathrm{A} \\
\mathrm{gcc}\end{array}$ & $\begin{array}{l}\mathrm{V} \\
\text { gtc }\end{array}$ & & $\begin{array}{c}\mathrm{Y} \\
\mathrm{tac}\end{array}$ & $\begin{array}{l}\mathrm{C} \\
\text { tgt }\end{array}$ & $\begin{array}{c}\text { A } \\
\text { gct }\end{array}$ \\
\hline AJ851868 IGHV14-3*02 & --- & --- & --- & --- & --- & --- & --- & --- & --- & --- & --- & -- & --- & --- & --- \\
\hline pComb3H-SS-WW6B7 & $\begin{array}{c}\mathrm{R} \\
\text { aga }\end{array}$ & $\underset{t c g}{S}$ & $\begin{array}{l}\text { I } \\
\text { atc }\end{array}$ & $\begin{array}{c}Y \\
\text { tac }\end{array}$ & $\begin{array}{c}\mathrm{Y} \\
\text { tat }\end{array}$ & $\begin{array}{c}\text { G } \\
\text { ggt }\end{array}$ & $\begin{array}{c}F \\
t t c\end{array}$ & $\stackrel{\text { A }}{\text { gcc }}$ & $\underset{\mathrm{cac}}{\mathrm{H}}$ & $\begin{array}{c}Y \\
c \text { tac }\end{array}$ & $\begin{array}{l}F \\
t t t\end{array}$ & $\begin{array}{c}\text { D } \\
\text { gac }\end{array}$ & $\begin{array}{l}Y \\
\text { tac }\end{array}$ & $\begin{array}{l}W \\
\text { tgg }\end{array}$ & $\begin{array}{l}\text { G } \\
\text { ggc }\end{array}$ \\
\hline
\end{tabular}

(b)

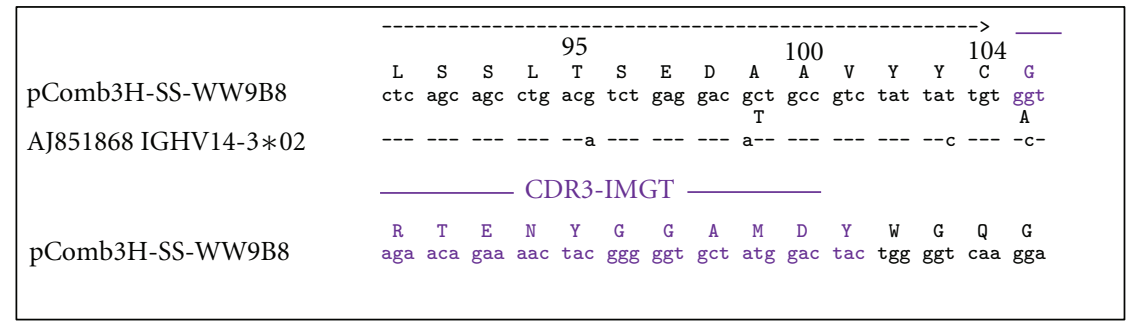

(c)

FIgure 2: CDR3 sequences of anti-MICA monoclones. Nucleotide and deduced amino acid sequences of V segment of (a) WW2G8, (b) WW6B7, and (c) WW9B8 compared with the reference sequence to identify CDR3. This region was randomly mutated by PCR mutagenesis creating a library of mutated CDR3 on WW2G8, WW6B7, and WW9B8 frameworks, and the high affinity phages were reselected by biopanning. Dashes indicate identical nucleotides.

$1 \mathrm{xPBS}$ and stained with $5 \mu \mathrm{L}$ of fluorescein isothiocyanate (FITC) labeled anti-M13 bacteriophage G8P capsid antibody (ABIN125966 lot\# 109131 antibodies-online GmbH, USA) for $1 \mathrm{hr}$ on ice, followed by $1 \mathrm{x}$ wash with $1 \%$ bovine serum albumin in 1xPBS. Cells bound phage-displayed anti-MICA Fab was analyzed by BD FACS Canto II (BD, USA). 293T cells $\left(2 \times 10^{5}\right.$ cells $)$ were incubated with VCSM13 as negative control.

\section{Results and Discussions}

3.1. Construction of Fab from Anti-MICA Monoclones. VH and VL of anti-MICA monoclones (WW2G8, WW6B7, and WW9B8) were amplified by PCR and cloned into pComb3H-SS. The heavy and light chain fragments were approximately $660 \mathrm{bp}$ (Figure 1 ). These clones were transformed into E. coli (Xl-1blue) by the standard heat shock method, rescued by M13 helper phages and isolated by biopanning against MICA antigens. These clones were validated by ELISA to ensure that they were carrying Fab binding to MICA. Sequences of CDR3 derived from WW2G8, WW6B7, and WW9B8 are shown in Figure 2. Mutagenesis primers of CDR3 (Table 1) were designed based on these sequences. Finally, these clones were used as templates for mutagenesis of HCDR3.

3.2. Affinity Maturation of Phages Displaying Anti-MICA Binding Activity. Ten rounds of biopanning were performed to select the positive phages with increased binding activities against MICA. Antigen-binding activities of the phages from the third to ten rounds of panning were analyzed by indirect ELISA, which showed the enrichment of binding activities indicated by greater OD during the panning cycle. Positive clones with high activities markedly predominated at the 7 th round of selection (Figure 3 ). Apparently, further rounds of biopanning did not much increase the binding activities. Sixty one clones of phages displaying mutated anti-MICA antibodies (WW9B8, WW6B7, and WW2G8) were randomly picked from the pool phages of the 7 th round selection and tested for their MICA binding activities by indirect ELISA. Among them, we obtained five clones that exhibited 3-7-folds higher antigen-binding activities indicated by OD (phage-display mutant Fab WW2G8.2, phage-display mutant Fab WW2G8.13, phagedisplay mutant Fab WW6B7.12, phage-display mutant Fab WW9B8.1, and phage-display mutant Fab WW9B8.21) (Figure 4 ). The comparative activities of phage-display Fab 


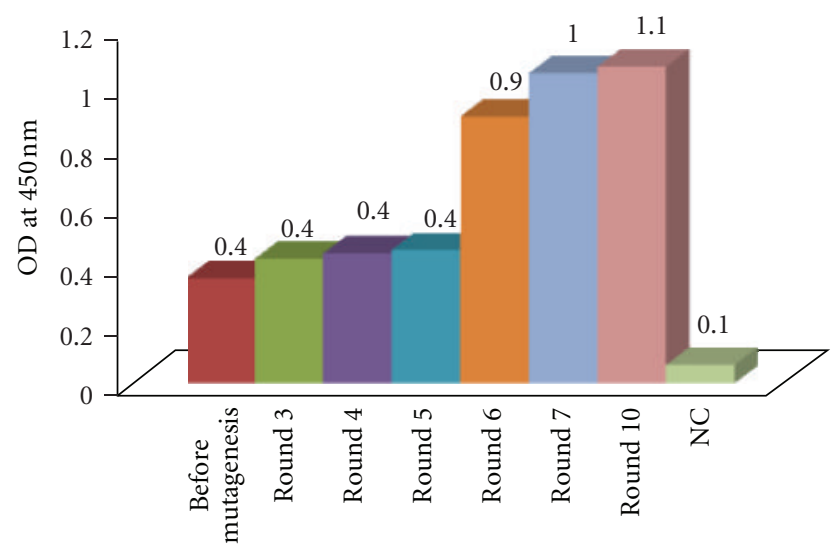

(a)

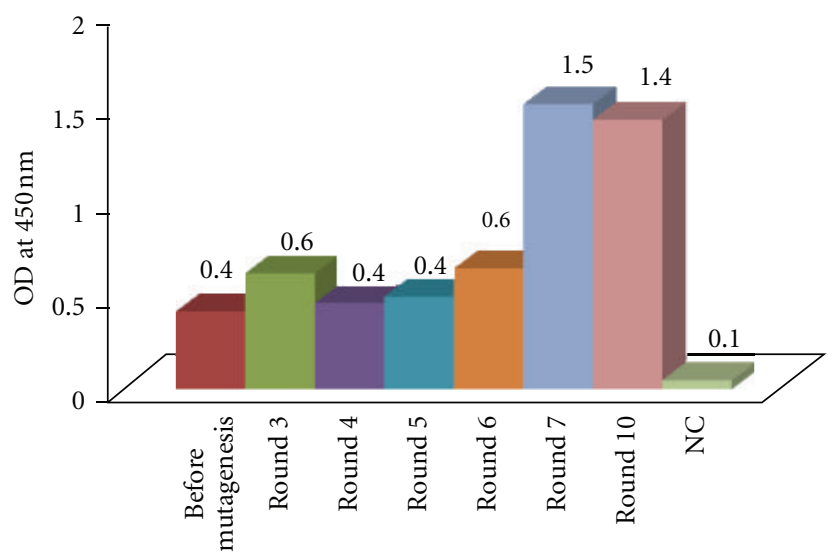

(b)

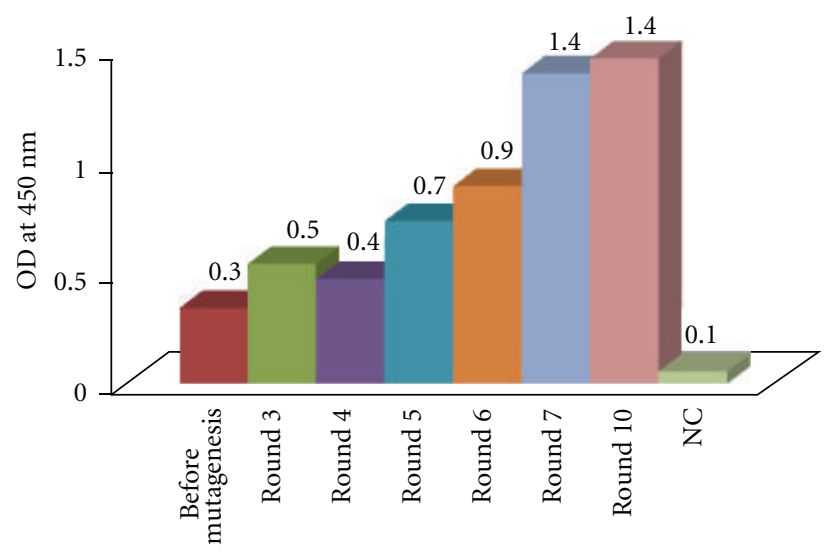

(c)

FIGURE 3: Affinity maturation of phages displaying Fab HCDR3 mutant of anti-MICA antibodies. After random mutagenesis of CDR3 of 3 anti-MICA phage display clones, they were selected against MICA by biopanning of ten rounds. Phage pools $\left(10^{8} \mathrm{pfu}\right)$ of each round were tested for MICA binding by ELISA compared to the corresponding phages before mutagenesis $\left(10^{8} \mathrm{pfu}\right)$. VCSM13 (10 ${ }^{8}$ pfu) was used as a negative control (NC). Comparative OD values of each round were shown in (a) WW2G8, (b) WW6B7, and (c) WW9B8. Apparently, the OD's of selections after 7 th round were not much increased. The final OD values were increased by $3-5$-folds compared to the original clones. Thus, the pool phages of the 7 th round were used to isolate individual phages with high binding activities indicated by OD values.

WW9B8, phage-display mutant Fab WW9B8.1, and phagedisplay mutant Fab WW9B8.21 were also determined by flow cytometry using $293 \mathrm{~T}$ cells expressing MICA. All three phage clones could detect MICA proteins on 293T. The phagedisplay mutant Fab WW9B8.1 and phage-display mutant Fab WW9B8.21 had higher activities against MICA proteins than the original phage-display Fab WW9B8 (Figure 5) corresponding to their activities determined by indirect ELISA. These two phage clones had the highest OD of all 5 clones isolated.

\section{Discussion}

The conventional hybridoma method of antibody production is time consuming and laborious. Previously, we have produced some monoclonal antibodies against MIC [17]. Anticancer drugs conjugated directly to antibodies are not efficient, and only a limited number of drug molecules could be conjugated leading to low drug carrier. In order to produce MICA-targeted anticancer drug, monoclonal antibodies against MICA were cloned and displayed on filamentous bacteriophages. Unlike drug conjugated to an antibody, a large amount of anticancer drug can be easily conjugated by chemical linking to pVIII which exists 2,700 copies as major-phage-coated proteins per a phage particle [21]. Anticancer-drug-conjugated bacteriophages have been used successfully with a potentiator factor of over 1,000 compared to free drug [22]. The studies of phages as targeted drug carriers were mostly in vitro. The in vivo study was only in a murine model [23]. Immunogenicity of bacteriophages in drug administration would be challenging and required further in vivo studies, especially in human.

The role of NKG2D receptor and ligands in immune responses against cancer is well established and has been exploited as approaches for cancer immunotherapy. These include the induction of anti-MICA to stimulate antitumor cytotoxicity [24], therapeutic DNA-based vaccine of NKG2D ligands and tumor antigens [25], and the generation of $\mathrm{T}$ cells with chimeric NKG2D receptors directly activated by ligand engagement $[26,27]$. However, the approaches 


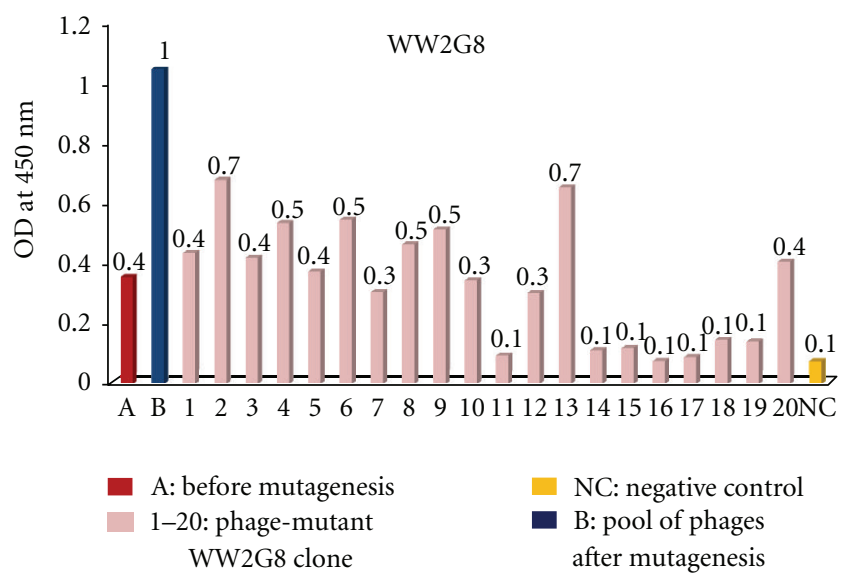

(a)

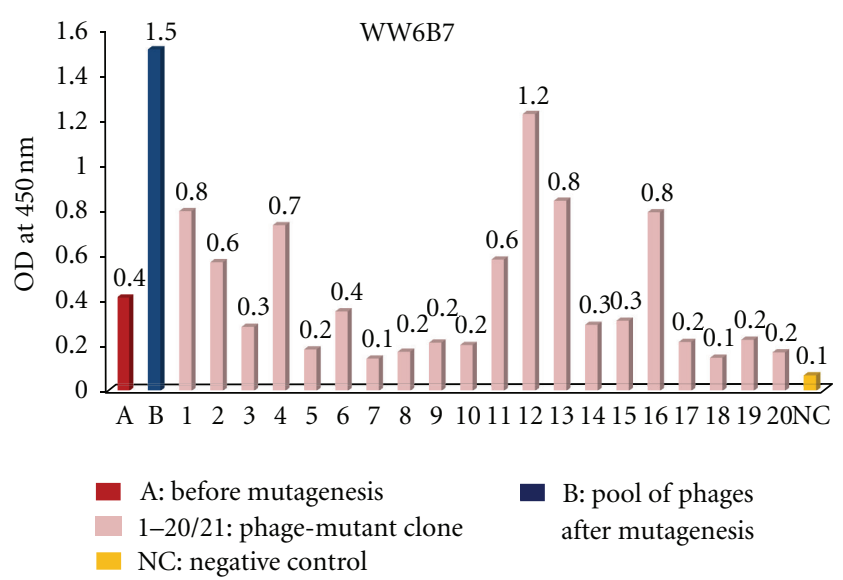

(b)

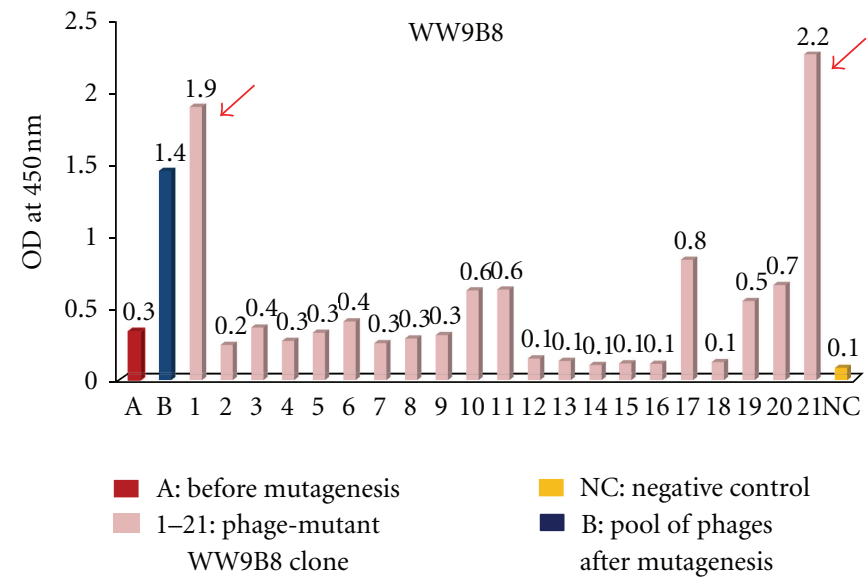

(c)

FIGURE 4: Isolation of individual mutant clones carrying improved binding activities of phages displaying anti-MICA. Individual clones $\left(10^{8} \mathrm{pfu}\right)$ isolated from the 7th round of biopanning were tested for MICA binding by ELISA. WW2G8, WW6B7, and WW9B8 mutants were shown in (a), (b), and (c), respectively. VCSM13 ( $\left.10^{8} \mathrm{pfu}\right)$ was used as a negative control. It should be noted that most of isolated clones had OD less than the pool phages with the exception of WW9B8. There were two WW9B8 mutants (red arrow) with OD values of 6 and 7 times higher than that of the pool phages, respectively.

employing drug conjugated to anti-NKG2D ligands have not been reported.

Apparently, the original anti-MICA displayed phages had less activities to detect MICA compared to monoclonal antibodies (Figure 5). It has been shown that mutations of HCDR3 of antibodies could allow antibodies to improve binding activity and specificity [21]. Thus, we performed in vitro affinity maturation by randomly mutating CDR3 which is one of the antigen-binding domains and selected for phages with higher activities by several rounds of biopanning. According to our data, at least seven rounds of selections would be needed for maximal enhancement. Normally, in other studies the panning was performed for only $3-5$ rounds $[18,28]$ resulting in a few positive antibody clones. Additional rounds of panning of up to ten rounds did not increase the collective OD. Among 61 individual clones of mutants, we obtained only fives clones $(8.19 \%)$ that had high binding activities. Two mutant clones with highest activities were characterized by flow cytometry (Figure 5) and sequencing (data not show). These phage clones were able to recognize MICA in a native form according to positive results obtained by both indirect ELISA and flow cytometry. To further characterize the high activities clones, competitive ELISA will be performed as well as testing against allelic MICA proteins to determine whether these clones have any limitation in detecting diverse MICA alleles.

In conclusion, phage display technology is an effective method to generate and isolate high affinity monoclonal antibodies against specific antigens. Phages displaying antiMICA with high binding activities have been established. By conjugation with anticancer drug such as doxorubicin or 5FU, these reagents would have high potential to develop targeted therapy against cancer cells expressing MICA proteins.

\section{Acknowledgments}

This work was supported by the Higher Education Research Promotion and National Research University Project of 


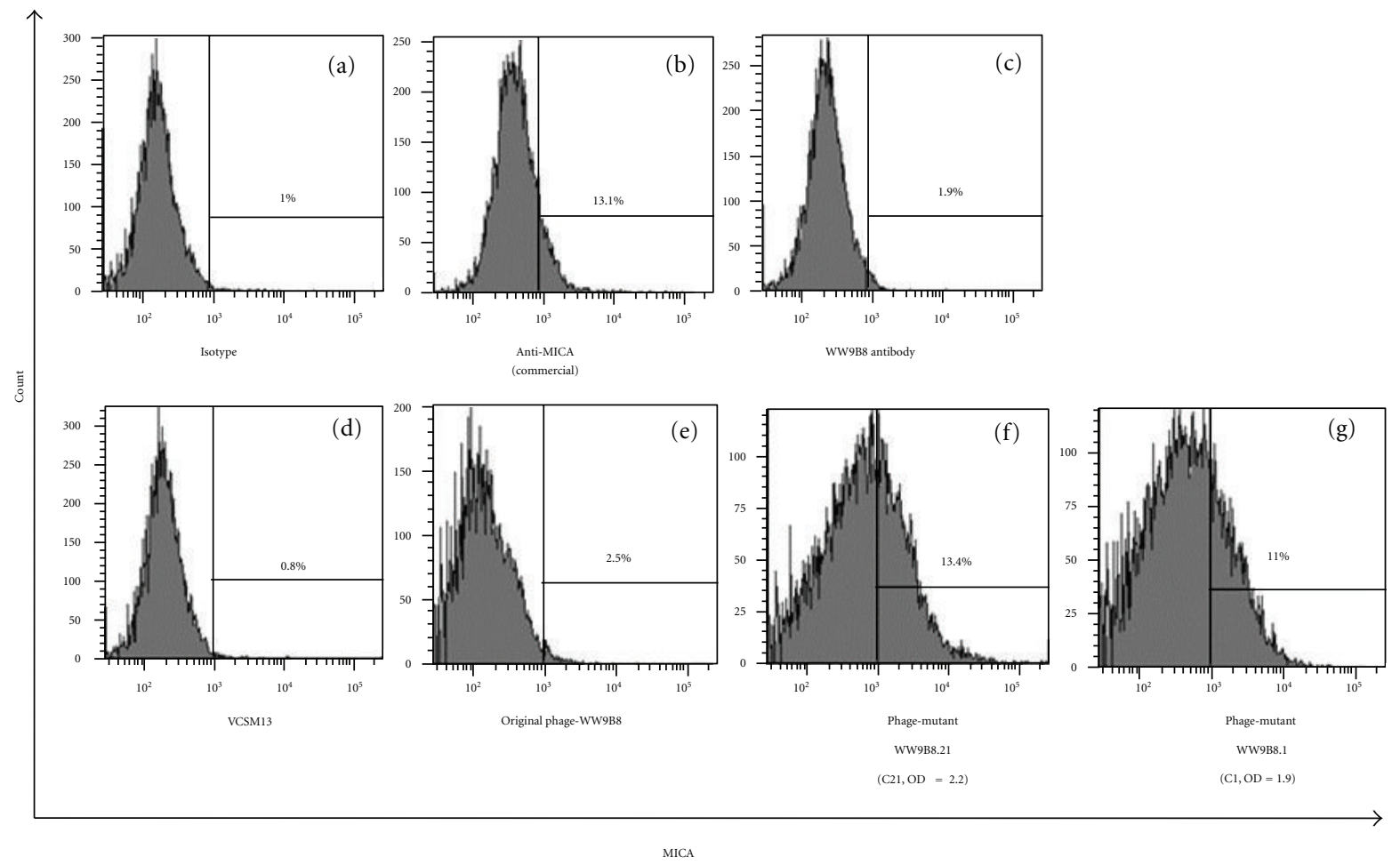

FIGURE 5: Characterization of anti-MICA displayed phages against MICA-positive cells by flow cytometry. $293 \mathrm{~T}$ cells $\left(2 \times 10^{5}\right)$ were incubated with (a) mouse IgG1 isotype control PE (b) anti-human MICA antibody PE, (c) anti-MICA WW9B8 and followed by goat $\mathrm{F}\left(\mathrm{ab}^{\prime}\right)_{2}$ anti-mouse IgG-PE. (d, e, f, g) 293T cells $\left(2 \times 10^{5}\right)$ were incubated with VCSM13 as negative control, original phages displaying Fab anti-MICA antibody (WW9B8), phages displaying mutant Fab WW9B8.21, phages displaying mutant Fab WW9B8.1 and followed by monoclonal antibody to M13 filamentous phage-FITC. The activities of phages displaying mutant WW9B8 are higher than the original phages carrying anti-MICA, WW9B8.

Thailand, Office of the Higher Education Commission, through the Center of Excellence in Specific Health Problems in Greater Mekong Subregion Cluster (SHeP-GMS), Project no. NRU 542022 and the Centre for Research and Development of Medical Diagnostic Laboratories (CMDL), Khon Kaen University (KKU). A. Phumyen is holding a Ph.D. scholarship (H-2533-Ph.d-04) supported by KKU through SHeP-GMS.

\section{References}

[1] C. Leelayuwat, D. C. Townend, M. A. Degli-Esposti, L. J. Abraham, and R. L. Dawkins, "A new polymorphic and multicopy MHC gene family related to nonmammalian class I," Immunogenetics, vol. 40, no. 5, pp. 339-351, 1994.

[2] S. Bahram, M. Bresnahan, D. E. Geraghty, and T. Spies, "A second lineage of mammalian major histocompatibility complex class I genes," Proceedings of the National Academy of Sciences of the United States of America, vol. 91, no. 14, pp. 6259-6263, 1994.

[3] D. Cosman, J. Müllberg, C. L. Sutherland et al., "ULBPs, novel MHC class I-related molecules, bind to CMV glycoprotein UL16 and stimulate NK cytotoxicity through the NKG2D receptor," Immunity, vol. 14, no. 2, pp. 123-133, 2001.

[4] M. Radosavljevic, B. Cuillerier, M. J. Wilson et al., "A cluster of ten novel MHC class I related genes on human chromosome 6q24.2-q25.3," Genomics, vol. 79, no. 1, pp. 114-123, 2001.
[5] A. V. Romphruk, T. K. Naruse, A. Romphruk et al., "Diversity of MICA (PERB11.1) and HLA haplotypes in Northeastern Thais," Tissue Antigens, vol. 58, no. 2, pp. 83-89, 2001.

[6] A. V. Romphruk, A. Romphruk, T. K. Naruse et al., "Polymorphisms of NKG2D ligands: diverse RAET1/ULBP genes in Northeastern Thais," Immunogenetics, vol. 61, no. 9, pp. 611617, 2009.

[7] S. Rareongjai, A. Romphruk, A. V. Romphruk, A. Sakuntabhai, and C. Leelayuwat, "Linkage disequilibrium of polymorphic RAET1 genes in Thais," Tissue Antigens, vol. 76, no. 3, pp. 230235, 2010.

[8] J. Wu, Y. Song, A. B. H. Bakker et al., "An activating immunoreceptor complex formed by NKG2D and DAP10," Science, vol. 285, no. 5428, pp. 730-732, 1999.

[9] S. Gilfillan, E. M. Ho, M. Cella, W. M. Yokohama, and M. Colonna, "NKG2D recriuts two distinct adapters to trigger NK cell activation and costimulation," Nature Immunology, vol. 3, no. 12, pp. 1150-1155, 2002.

[10] V. Groh, R. Rhinehart, H. Secrist, S. Bauer, K. H. Grabstein, and T. Spies, "Broad tumor-associated expression and recognition by tumor-derived $\gamma \delta$ T cells of MICA and MICB," Proceedings of the National Academy of Sciences of the United States of America, vol. 96, no. 12, pp. 6879-6884, 1999.

[11] V. Groh, R. Rhinehart, J. Randolph-Habecker, M. S. Topp, S. R. Riddell, and T. Spies, "Costimulation of $\operatorname{CD} 8 \alpha \beta$ T cell by NKG2D via engagement by MIC induced on virus-infected cells," Nature Immunology, vol. 2, no. 3, pp. 255-260, 2001. 
[12] H. Das, V. Groh, C. Kuijl et al., "MICA engagement by human $\mathrm{V} \gamma 2 \mathrm{~V} \delta 2 \mathrm{~T}$ cells enhances their antigen-dependent effector function," Immunity, vol. 15, no. 1, pp. 83-93, 2001.

[13] M. Champsaur and L. L. Lanier, "Effect of NKG2D ligand expression on host immune responses," Immunological Reviews, vol. 235, no. 1, pp. 267-285, 2010.

[14] S. J. Burgess, K. Maasho, M. Masilamani, S. Narayanan, F. Borrego, and J. E. Coligan, "The NKG2D receptor: immunobiology and clinical implications," Immunologic Research, vol. 40, no. 1, pp. 18-34, 2008.

[15] R. A. Eagle and J. Trowsdale, "Promiscuity and the single receptor: NKG2D," Nature Reviews Immunology, vol. 7, no. 9, pp. 737-744, 2007.

[16] V. Groh, J. Wu, C. Yee, and T. Spies, "Tumour-derived soluble MIC ligands impair expression of NKG2D and T-cell activation," Nature, vol. 419, no. 6908, pp. 734-738, 2002.

[17] W. Wongsena, G. Sconocchia, H. S. Cho et al., "Production and characterization of monoclonal antibodies against major histocompatibility complex class I chain-related gene A," Tissue Antigens, vol. 72, no. 5, pp. 431-440, 2008.

[18] C. F. Barbas III, "Recent advances in phage display," Current Opinion in Biotechnology, vol. 4, no. 5, pp. 526-530, 1993.

[19] K. Jury, P. Söhnlein, M. Vogel, and W. Richter, "Isolation and functional characterization of recombinant GAD65 autoantibodies derived by IgG repertoire cloning from patients with type 1 diabetes," Diabetes, vol. 50, no. 9, pp. 1976-1982, 2001.

[20] A. Jumnainsong, P. Jearanaikoon, S. Khahmahpahte et al., "Associations of MICB with cervical cancer in north-eastern thais: identification of major histocompatibility complex class I chain-related gene B motifs influencing natural killer cell activation," Clinical and Experimental Immunology, vol. 153, no. 2, pp. 205-213, 2008.

[21] S. A. Overman, M. Tsuboi, and G. J. Thomas Jr., "Subunit orientation in the filamentous virus Ff(fd, f1, M13)," Journal of Molecular Biology, vol. 259, no. 3, pp. 331-336, 1996.

[22] H. Bar, I. Yacoby, and I. Benhar, "Killing cancer cells by targeted drug-carrying phage nanomedicines," BMC Biotechnology, vol. 8, article 37, 2008.

[23] L. Vaks and I. Benhar, "In vivo characteristics of targeted drugcarrying filamentous bacteriophage nanomedicines," Journal of Nanobiotechnology, vol. 9, article 58, 2011.

[24] M. Jinushi, F. S. Hodi, and G. Dranoff, “Therapy-induced antibodies to MHC class I chain-related protein a antagonize immune suppression and stimulate antitumor cytotoxicity," Proceedings of the National Academy of Sciences of the United States of America, vol. 103, no. 24, pp. 9190-9195, 2006.

[25] H. Zhou, Y. Luo, J. F. Lo et al., "DNA-based vaccines activate innate and adaptive antitumor immunity by engaging the NKG2D receptor," Proceedings of the National Academy of Sciences of the United States of America, vol. 102, no. 31, pp. 10846-10851, 2005.

[26] T. Zhang, A. Barber, and C. L. Sentman, "Generation of antitumor responses by genetic modification of primary human T cells with a chimeric NKG2D receptor," Cancer Research, vol. 66, no. 11, pp. 5927-5933, 2006.

[27] A. Barber, T. Zhang, and C. L. Sentman, "Immunotherapy with chimeric NKG2D receptors leads to long-term tumorfree survival and development of host antitumor immunity in murine ovarian cancer," The Journal of Immunology, vol. 180, no. 1, pp. $72-78,2008$.
[28] G. H. Yang, S. O. Yoon, M. H. Jang, and H. J. Hong, "Affinity maturation of an anti-hepatitis B virus PreS1 humanized antibody by phage display," The Journal of Microbiology, vol. 45, no. 6, pp. 528-533, 2007. 


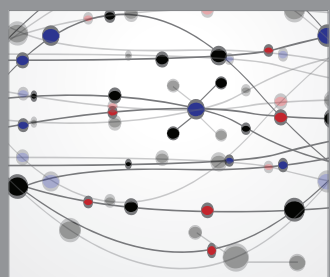

The Scientific World Journal
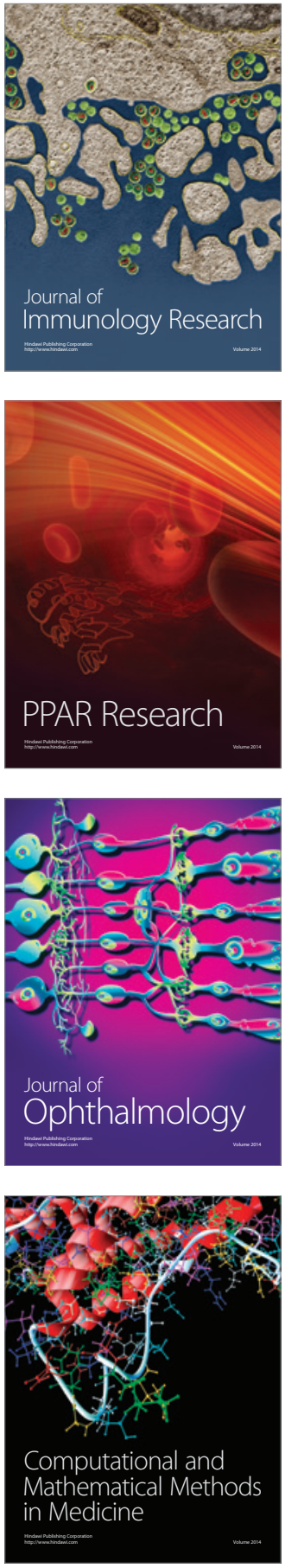

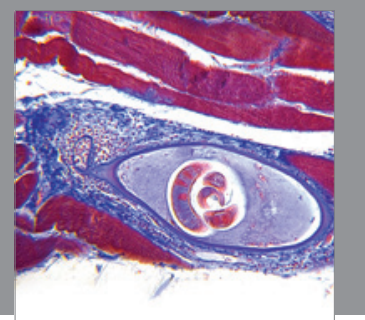

Gastroenterology

Research and Practice
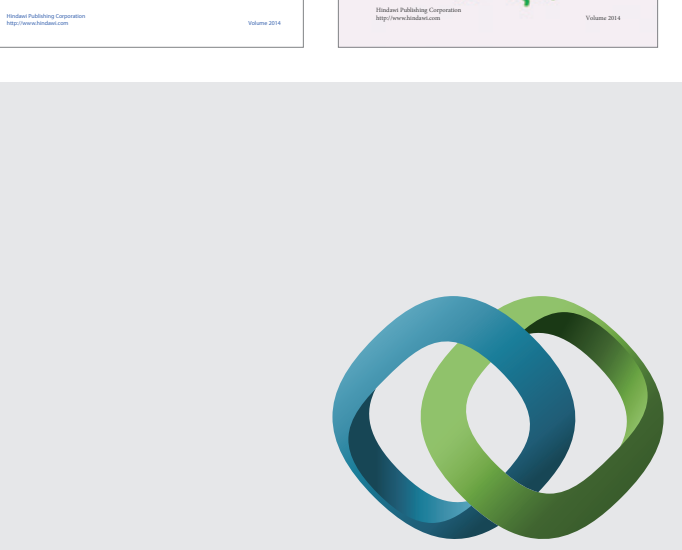

\section{Hindawi}

Submit your manuscripts at

http://www.hindawi.com
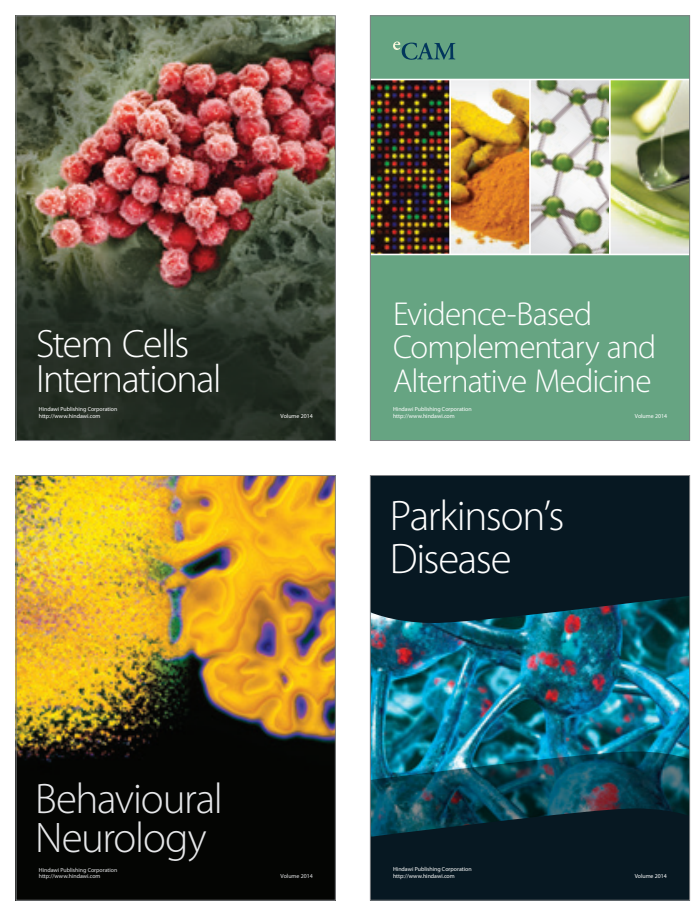

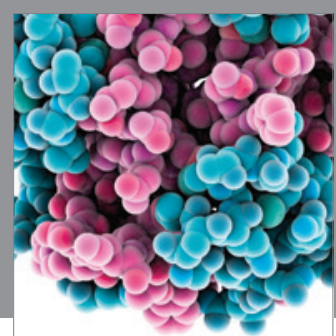

Journal of
Diabetes Research

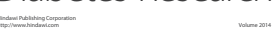

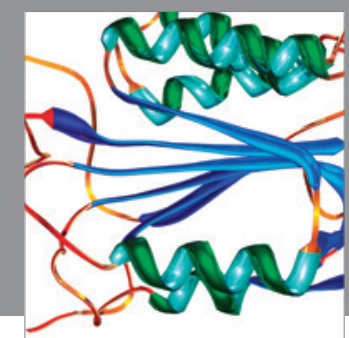

Disease Markers
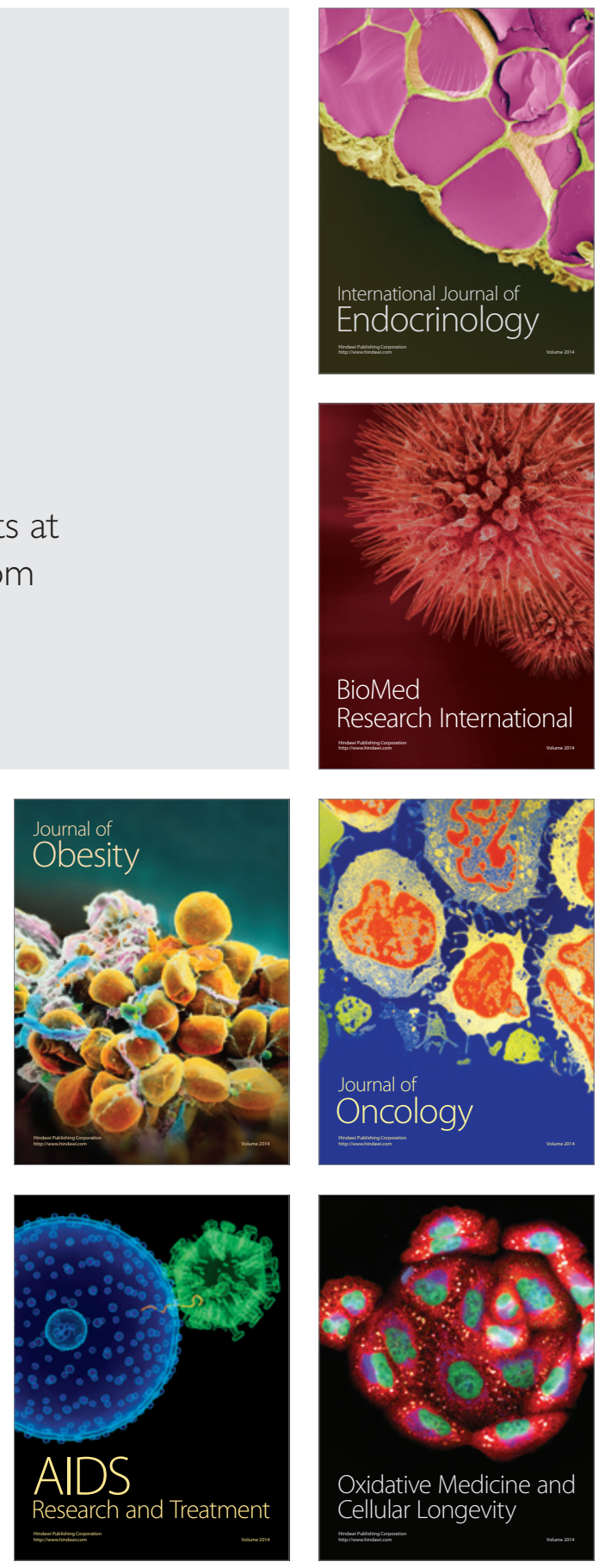